\title{
Research on Location of Line Spectrum of Underwater Target Based on Passive Synthetic Aperture Method
}

\author{
Zhu Sanwen ${ }^{\text {a }}$, Wang Huakui, Zhang Xuebo \\ Sci. \& Tech. on Underwater Acoustic Antagonizing Laboratory, Zhanjiang, China \\ asanwenz@sina.com
}

\begin{abstract}
Keywords: Line Spectum, Passive Synthetic Aperture, Location, Doppler Shift, Azimuth Compressing Function
\end{abstract}

\begin{abstract}
Considering the location demand of radiated noise source in underwater target, the method of location of line spectrum of radiated noise based on passive synthetic aperture method is presented. Line spectrum signal is received using single hydrophone, and azimuth sampling rate depends on the azimuth doppler band, and the received data of one-dimension is transformed into data of two-dimension. Azimuth compressing function is configured based on the range curve and the frequency of line spectrum, image of line spectrum is derived in frequeny-azimuth domain. Simulated results show that line spetrum of radiated noise can be located and distinguished, and the resolution is dependent on the azimuth doppler band.
\end{abstract}

\section{Introduction}

Sound source location is an important task in underwater target radiated noise measurement, and it is also a difficult problem to date. To locate the line spectrum in underwater target can provide technical support for vibration damping, noise reduction, and maintenance which helps to improve battle effectiveness and the existing ability for the underwater target[1-2].

Owing to the relative motion between the underwater target and the measuring hydrophone, doppler shift exist in the line spectrum[3]. Processing the azimuth dopper signal using the same method as in SAR and SAS can determine the azimuth location of the line spectrum in underwater target. In the thesis, localization of the line spectrum using the passive sythetic aperture method is investigated, and simulation tests show the effectiveness of the proposed method.

\section{Line spectum of the radiated noise.}

Underwater target radiated noise consists of line spectrum and continuous spectrum, and can be expressed by the summation of the two parts[4]. Line spectrum whose variation is less than $1 \mathrm{~Hz}$ during hundreds of seconds is caused by the rotation of the propeller and the repeated motion of reciprocating machinery, and the amplitude of line spectrum is $5 \sim 7 \mathrm{~dB}$ higher or even more than that of continuous spectrum. The space-time coherence of sea envrionment is the key factor that affects the formation of the synthetic aperture. Researchs show that the space coherence length reaches 300 wavelengths or so for line spectrum signal during $50 \sim 1500 \mathrm{~Hz}$, and the time coherence length of CW is not less than several minutes or even tens of minutes. Therefore, the space-time stability of line spectrum provides the feasibility of forming the synthetic aperture.

\section{Analysis of the synthetic aperture for line spectrum of the radiated noise of the underwater target.}

(a) Principle of passive synthetic aperture

In passive synthetic aperture processing, the parameters of the target are estimated by virtue of the radiated signal of the underwater target received by the virtual array[5-7]. As shown in Fig.1, underwater target moves in a strait line, and the nearest distance between the measuring hydrophone and the underwater target's ideal track is R0, and the radiated noise signal is sampled by the 
measuring hydrophone. To apply synthetic aperture technology, we can consider the problem in a converse way, the underwater target is stationary, and the measuring hydrophone is moving toward and outward the underwater target at constant speed $v$ in a stait line while sampling the radiated signal. Thus, a series of virtual hydrophones are formed along the track of the underwater target when the underwater target is moving, and the resolution and gain which are equal to those of real aperture of the same size can be attained.
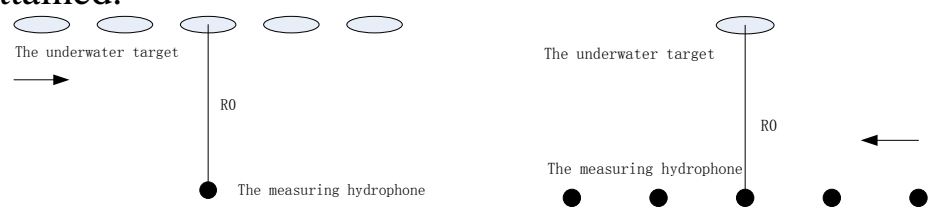

Fig.1 Illustration of passive synthetic aperture.

(b) Azimuth sampling rate of passive synthetic aperture

There exists doppler shift fd in received signal due to the motion of the underwater target, and it can be expressed as follows:

$$
f_{d}=f_{0}\left(1 \pm \frac{v \sin \theta}{c}\right)
$$

Where $\theta$ is the angle between line of sight and the moving direction of the underwater target, and doppler shift $f_{d}$ varies with $\theta$ and azimuth time ta. In Fig. 2, the relation between doppler shift $f_{d}$ and azimuth time ta is depicted.

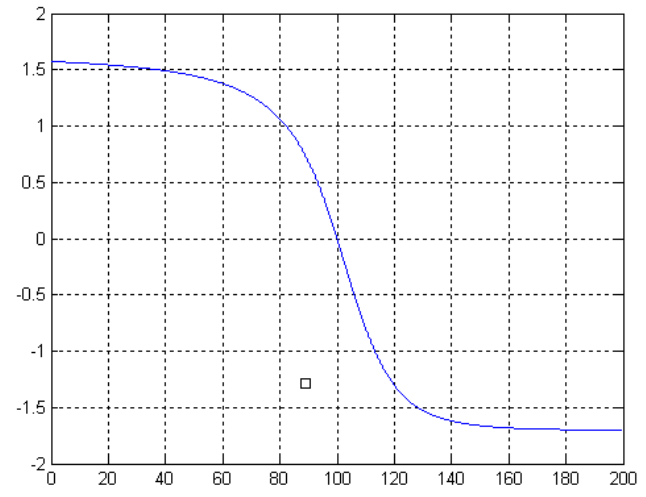

Fig. 2 Relation between doppler shift $f_{d}$ and azimuth time ta

For mono-frequency sigal with fequency $f_{0}$, doppler band $\mathrm{B}$ is less than $2 \mathrm{v} / \lambda$ in which $\mathrm{v}$ denotes the speed the underwater target. In relation of the sampling theory, to sample the signal without distortion requests that the sampling rate $f s$ be more than two times $f c$ which is the highest frequency of the signal. In our simulation test, the azimuth sampling rate prf is set as $4 v / \lambda$ which is over two times of doppler band B.

(c) Segment of the received signal and phase compensation

Having determined the azimuth samplingt rate $p r f$, the azimuth sampling period pri can be defined as $1 / p r f$. The system sampling rate is fs, and the number of azimuth sampling point $\mathrm{Na}$ can be defined as follows:

$$
\mathrm{Na}=\text { floor(T/pri) }
$$

Where $\mathrm{T}$ is the duration time of the received signal $\mathrm{s}(\mathrm{t})$, and $\mathrm{s}(\mathrm{n})$ is the discrete form of $\mathrm{s}(\mathrm{t})$. The one-dimension received signal $s(n)(n=1, \cdots N)$ can be divided into Na segments which length is equal to $\mathrm{Nr}$ and two-dimension signal ss(na, $\mathrm{nr})(\mathrm{na}=1, \cdots, \mathrm{Na}, \mathrm{nr}=1, \cdots, \mathrm{Nr})$ is constructed based on the $\mathrm{Na}$ segments.

In order to ensure the coherence of the different azimuth time data ss(na,:), the time delay error $\Delta_{\mathrm{n}} \mathrm{t}$ induced by the segment of the data must be compensated.

$$
\Delta_{n} t=(n-1) \cdot\left(p r i-n \cdot t_{s}\right)
$$

For narrow-band signal, compensation can be implemented by phase multiplication in each azimuth time data ss(na,:). For broad-band signal, the process must be carried out by interpolation. 
(d) Resolution in azimuth and range

Resoluton $\rho_{a}$ in azimuth of SAR/SAS can be expressed as following:

$$
\rho_{a}=k \frac{\lambda}{D}
$$

Where $k$ is a constant, and $\lambda$ is the wavelength of the mono-frequency signal, and D denotes the synthetic aperturelength. Azimuth resoluton $\rho_{a}$ depends on the wavelength $\lambda$ and the length of synthetic aperture $\mathrm{D}$. The longer of the wavelength $\lambda$ and the shorter of the length of synthetic aperture $\mathrm{D}$, azimuth resolution $\rho_{a}$ is lower, vice versa.

In our simulation, the same synthetic aperture length is set for all line spectrum signal. That is to say, for lower frequency signal, lower azimuth resolution can be obtained, and high azimuth resolution can be acquired for higher frequency signal. In practical working process, different line spectrum signal have different synthetic aperture length.

For line spectrum signal, there is no resolution in range. In our working case, the range between the underwater target moving at constant speed in a strait line and the measuring hydrophone can be derived by the navigation devices equiped.

(e) Configuration of azimuth compressing function

To compress in azimuth, azimuth compressing function can be defined as:

$$
\begin{aligned}
H & =\exp \left(-j \cdot k_{n} \cdot R\left(t_{a}\right)\right) \\
R\left(t_{a}\right) & =\sqrt{\left(x a\left(t_{a}\right)-x_{0}\right)^{2}+\left(y a\left(t_{a}\right)-y_{0}\right)^{2}}
\end{aligned}
$$

Where $k_{n}=2 \pi f_{n} / C$ is wave number of the line spectrum signal, and $f_{n}$ is the frequency of the line spectrum signal, and $t_{a}$ is azimuth time. $\left[x a\left(t_{a}\right), y a\left(t_{a}\right)\right]$ is the azimuth location of the measuing hydrophone at time $t_{a}$, and is 0 when there is no motion error. $\left[x_{0}, y_{0}\right]$ is the geometry center of the underwater target, and $x_{0}$ is zero, and $y_{0}$ is the nearest distance between the measuring hydrophone and the ideal track of the underwater target. From equation (7), $R\left(t_{a}\right)$ is the function of azimuth time $t_{a}$ which shows the variation of the doppler shift. Azimuth compression can be implemented according to equation (6) in time domain or frequency domain.

(f) Determination of the imaging plane

For different line spectrum signal, the azimuth loacation can be determined by azimuth compression. Being no resolution in range, imaging plane is $\mathrm{O}(f r, x a)$ where $f r$ denotes frequency in range. Through passive synthetic aperture processing, line spectrum signal of different frequency and azimuth location has different bright spots in $\mathrm{O}(f r, x a)$ imaging plane.

\section{Simulation}

In order to test the effectiveness of algorithm, simulaiton test is carried out in the thesis. Assumptions: The medium is homogenous, and the infulence of the noise is neglected. The parameters are set as Table.I

Table.I system paramenters

\begin{tabular}{|l|l|l|l|}
\hline Sampling rate & $6 \mathrm{kHz}$ & Synthetic aperture length & $200 \mathrm{~m}$ \\
\hline Veloctiy of the underwater target & $5 \mathrm{~m} / \mathrm{s}$ & $\mathrm{R} 0$ & $100 \mathrm{~m}$ \\
\hline Sound of speed in water & $1500 \mathrm{~m} / \mathrm{s}$ & & \\
\hline
\end{tabular}

The parameters of the five line spectrum signal are set as Tabel.II

Tabel.II target parameters

\begin{tabular}{|l|l|l|l|}
\hline $\mathrm{f} 1$ & $133 \mathrm{~Hz}$ & xa1 & $5 \mathrm{~m}$ \\
\hline $\mathrm{f} 2$ & $266 \mathrm{~Hz}$ & xa2 & $10 \mathrm{~m}$ \\
\hline $\mathrm{f} 3$ & $400 \mathrm{~Hz}$ & xa3 & $15 \mathrm{~m}$ \\
\hline $\mathrm{f} 4$ & $533 \mathrm{~Hz}$ & xa4 & $20 \mathrm{~m}$ \\
\hline $\mathrm{f} 5$ & $666 \mathrm{~Hz}$ & xa5 & $25 \mathrm{~m}$ \\
\hline
\end{tabular}




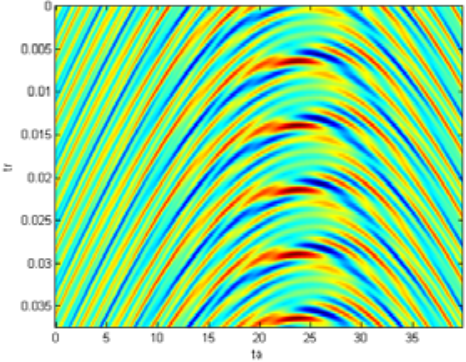

(a)

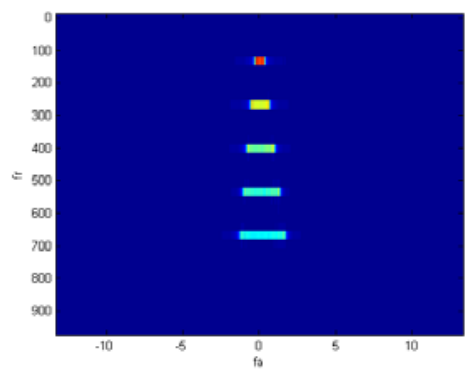

(c)

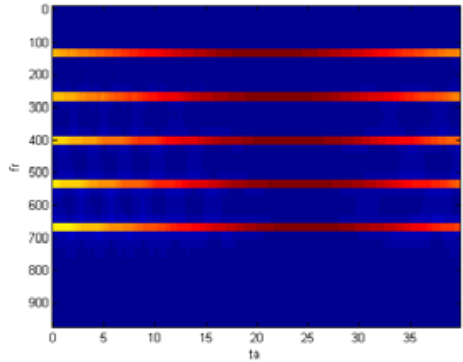

(b)

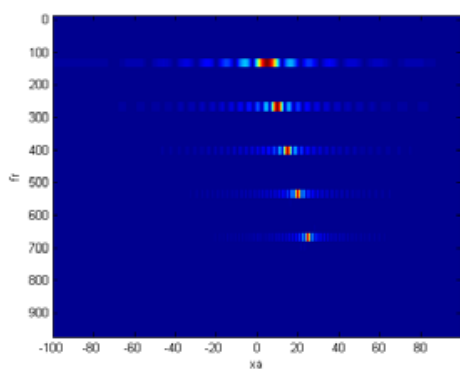

(d)

Fig.3 Simulation of location of line spectrum signal using passive synthetic aperture method.

Fig.3(a) is the received two-dimension signal ss(na,nr) containing 5 line spectrum signal in whicn xlabel denotes azimuth time ta and ylabel is range time tr. Each line spectrum signal has its own frequency and azimuth location. Fig.3(b) is the FFT transformation of ss(na,nr) in range, in which xlabel denotes azimuth time and ylabel is frequency in range. We can see 5 line spectum signal in Fig.3(b). Fig.3(c) is two-dimension FFT transformation of ss(na,nr), in which xlabel denotes frequency in azimuth and ylabel is frequency in range. In Fig.3(c), different line spectrum signal has different doppler band widths, and doppler centers are all zeros. Fig.3(d) is the result of azimuth compression, and the locations of the bright spots correspond well to the locations of the line spectrum signal.

\section{Conclusion}

Line spectum signal of the underwater target is located in azimuth using the received data by the measuring hydrophone based on passvie syntheti aperture method. Simulation results show that line spectrum signal of different frequency and azimuth location can be located and distinguished in azimuth, and there is no resolutin in range which meet the need of noise measurement.

\section{References}

[1]Wang Zhicheng,Cheng Zongj,Liu Wenshuai. Measurement and analysisof ship radiated noise[M]. Publishing company of defense industry, 2004.

[2]Zhang Linke, He Lin, Zhu Shijian. Research on identification of the main noise source of submarine[J]. Vibration and noise control. 2006(4):7-10.

[3]Wu Guoqing, Ma Li. Range Estimation based on doppler shift of multi line spectrum of radiated noise[J]. Journal of Acoustics, 2006(3), volume 31,issue 2,page:140-145.

[4]Urick. Principle of underwater acoustics[M]. Publishing company of Institute of Haerbin Ship and Engineering. 1990.

[5]C Yen, W Carey. Application ofsynthetic aperture processing to towed- array data[ J]. Journal of Acoustical Society America,1989, 86: 754-765.

[6]S. Stergiopoulos, E. H. Sullivan, Extended towed array processing by an overlap correlator, J. Acoust. Soc. Am., vol.86, pp. 158-171, 1989

[7]Stergiopoulos and Urban, A new passive synthetic aperture technique for towed arrays, IEEE Journal of Oceanic Engineering, Vol 17, No. 1, January 1992 